\title{
Implementation of Attendance System using Face Recognition and PCA
}

\author{
Sonali Patil \\ JSPM's Jayawantrao Sawant \\ College of Engineering, Pune
}

\author{
Avdhoot Gaikwad \\ JSPM's Jayawantrao Sawant \\ College of Engineering, Pune
}

\author{
Chetana Baviskar \\ JSPM's Jayawantrao Sawant \\ College of Engineering, Pune
}

\author{
Shrawani Bartakke \\ JSPM's Jayawantrao Sawant College of \\ Engineering, Pune
}

\author{
Shubham Kulkarni \\ JSPM's Jayawantrao Sawant College of \\ Engineering, Pune
}

\begin{abstract}
The prototype of an automated Online Biometric-enabled Class Attendance Register System is presented in this study (OBCARS). The technology is being planned and developed to address the problem of lost and/or shredded attendance record paper sheets in higher education classrooms. The system also seeks to provide a reliable and efficient class attendance tracking system that prevents students from imitating attendance markers and streamlines the calculation of students' attendance records. Both pragmatic biometric behaviourin contrast to previously poised data for a focus and an open-mindedness of computation are included in biometric appreciation. Estimated identical is required because to variances in biological features and deeds both within and among humans. It determines a student's attendance by their attendance in class. The technology willrecognize the student's face and save the response to the database automatically.
\end{abstract}

\section{Keywords}

Face Recognition, Attendance System, Python, Open CV, PCA, Deep Learning, Image Processing, Database.

\section{INTRODUCTION}

Face recognition is one of the few biometric technologies that manages to be both accurate and non-intrusive. Since the early 1970s, face recognition has captivated the interest of academics in fields ranging from security to image processing to computer vision. Face recognition has also proven useful in multimedia data processing. In the past,attendance was taken in the classroom manually using attendance registers issued to faculty members. It is, however, a time-consuming procedure. Furthermore, in a large classroom, checking eachstudentindividually to see if they are there or not is difficult. The proposed system illustrates how to use Java to use facial recognition to collect a student's attendance automatically, how to acquire the absence list and how to store the faces in a database. Detecting whether a person's face image matches any of the face images stored in a database. This challenge is challenging to address automatically due to the changes that various components, such as facial expression, ageing, and even lighting, can cause on the image. Although facial recognition is not the most reliable biometric method, it does have some advantages over the others. Security and access control, forensic medicine, police controls, and attendance management systems are just a few of the uses.

\section{LITERATURE SURVEY}

One of the most important aspects of school is keeping track of student attendance. One of the factors of student graduation is the student's attendance record. Attendance records that precisely recorded [5], [18] are required in practically every educational profession. As a result of the availability of various information and communication technology capabilities, procedures have evolved from manual to semiautomatic or automatic. The range of extant sensortechnologies allows for diverse combinations of processes to be produced by researchers and developers in a variety of ways, including the use of a camera [3]. Higher education uses a variety of methods to track student attendance, including manual, semi-automatic, and even technological systems [9]. At current time, various methods of recording student attendance are available and being developed; each system, of course, has advantages and disadvantages [25]. RFID [1], social media [10], barcodes, Bluetooth [2], fingerprints [17], and Near Field Communication (NFC) [4] are examples of technology used in this attendance system. This attendance system application was developed by a number of researchers and application developers. Attendance systems can be constructed in a variety of ways using various sensor technologies. One of the options used to track student attendance during class meetings is the use of facial recognition technology [18], [19]. Face capture using cameras and object protection systems that make up human faces are used in this technology [20]. After that, the system discusses each face's object, and each item is forecasted using a list of confirmed faces from the student's face library. The model of face recognition used in student participation systems in higher institutions is discussed in this paper. One possibility for colleges to use attendance systems on each campus is to apply facial recognition technology to the college attendance system. The term "computer vision" refers to the processing of digital images [6]. So that computers can see and understand objects in the same way as humans do [21]. As a result, the machine is capable of recognizing an object, making decisions, taking action, and counting the number of items [16]. Computer Vision [11] allows computers to learn from existing data and to learn on its own, allowing machines to see objects in the same way that people do [18]. Humans utilize computers in a variety of activities that need them to watch and pay attention to objects, until the machine is advanced enough to include student attendance systems in the learning processwith their lecturers. Machine learning has recently become one of the growing technologies [13] because to the presence and development of 
an accelerated Graphics Processing Unit (GPU). The use of fixed and moving graphics for various procedures required by people and their developers, in particular. Deep systematic learning [23], [14] is one of the technologies used. Deep learning's broad coverage is expanding, making it prominent in information processing via natural language processing, as well as capability extraction and visualization procedures. Deep Learning technology, especially its application to Computer Vision, has a strong ability to manage procedures linked to face identification. Convolutional Neural Networks (CNN) [24], [22] are one of the capabilities utilized for classifying objects in an image.The inclusion of numerous options, such as the success of the regional proposal approach and the RCNN [7], is helping to recognize objects in the form of images. [15] Through the real-time test of the algorithm, it fully meets the requirements of the attendance time in the class.

\section{PROBLEM STATEMENT}

To develop windows based prototype model for biometric attendance system using face recognition using python programming language.

\subsection{Goals and Objectives}

1. To implement biometric attendance system using face recognition.

2. To design a database and identify the person from pre-defined database.

3. To mark the attendance by comparing unique features of the face.

\subsection{Motivation}

Image processing, which deals with obtaining valuable information from a digital image, has played a significant part in the evolution of technology in recent years. It focuses on two objectives. Improvement of pictorial information for human interpretation.Image data processing for autonomous machine perception, including storage, transmission, and representation. With the introduction of smart phones and closed circuit television, individuals have begun to use picture capturing gadgets in ways they never have before. The manual attendance record system is inefficient, requiring extra time to organize records and determine each student's average attendance. As a result, a system to tackle the problem of student record organization and average attendance computation is required.

\section{PROPOSED SYSTEM}

The flow of the proposed system is as follows:

1. Prepare a training set of face database.

2. Input the face image to the machine learning model.

3. Removing the unnecessary part from the data which is called preprocessing.

4. Determine unique facial features using feature extraction technique.

5. Classify the data into different categories.

6. Store the result in the database.

7. Mark the attendance of the person.

Some of the strategies for keeping track of attendance are as follows:

Signature based system:

$$
\begin{array}{ll}
\text { 1. } & \text { Fingerprint based System } \\
\text { 2. } & \text { Iris Recognition } \\
\text { 3. } & \text { RFID based System } \\
\text { 4. } & \text { Face Recognition }
\end{array}
$$

Amongst the above techniques, Face Recognition is natural, easy to use and does not require aid from the test subject. It is a series of several related problems which are solved step by step:

1. To capture a picture and discern all the faces in it.

2. Concentrate on one face at a time and understand that even if a face isturned in a strange direction or in bad lighting, it is still the same person.

3. Determine various unique features of the face that can help in distinguishing it from the face of any other person. These characteristics could be the size eyes, nose, length of face, skin color, etc.

Compare these distinctive features of that face to all the faces of people we already know to find out the person's name. Our brain, as a human is made to do all of this automatically and instantaneously. Because computers are incapable of such high-level generalization, each stage of face recognition must be taught or programmed separately. Face recognition systems fall into two categories: verification and identification. Face verification is a $1: 1$ a face image is compared to a template face image whose identity is being claimed. On the contrary, face identification is a $1: \mathrm{N}$ problem that 


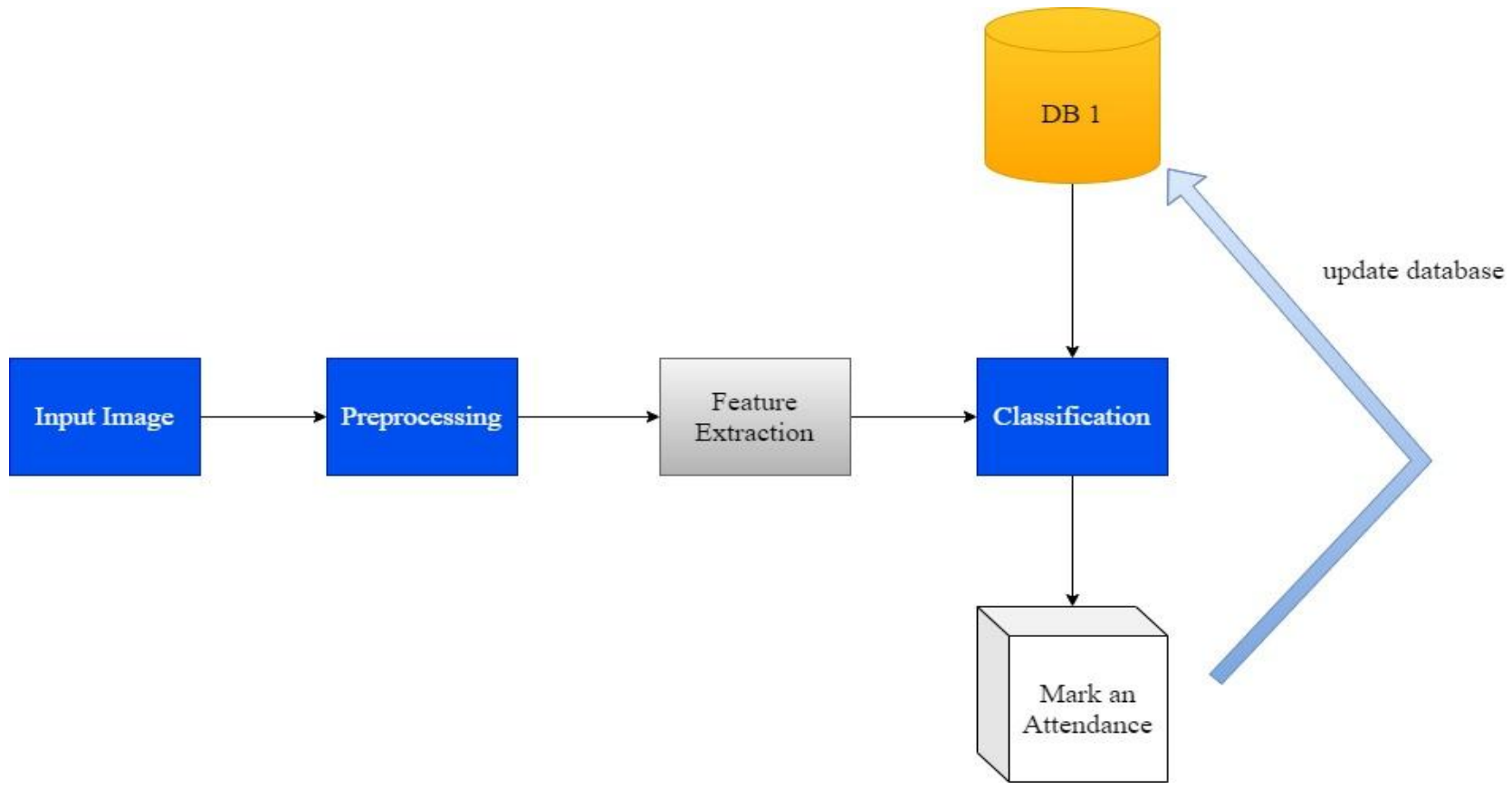

Fig.1: Proposed system architecture

\section{ALGORITHM}

The basic goal of principle component analysis (PCA) is to keep as much variance as feasible while reducing the dimensionality of a data set made up of numerous variables that are strongly or weakly related to one another. The same can be done by transforming the variables into a new set of variables called principal components (or simply PCs), which are orthogonal and organised in such a way that as we move down the list, the amount of variation retained in the original variables diminishes. As a result, the first principle component keeps the highest variance from the original components. The major components are orthogonal because they are the eigenvectors of a covariance matrix.

Steps of this proposed algorithm is as follows:

\section{A. Initialization process \\ B. Recognition process}

The initialization and recognition process involves the following operations:

1. Obtain the initial set of face photos, which is referred to as the training set.

2. Calculate the Eigen faces using only the highest eigenvalues from the training set. These $\mathrm{M}$ images define the face space. The Eigen faces can be updated or recalculated as new faces are encountered.

3. Calculate each known person's distribution in this M-dimensional space by projecting his or her face images onto this face-space.

4. By projecting the input image onto each of the faces, calculate a set of weights based on the input image.

5. Check to see if the image is close enough to an empty space to determine if it is a face at all (known or unknown).
6. If it's a face, decide if the pattern belongs to a recognized or unknown person.

\subsection{Pros}

1. Using this system it is easy to maintain attendance.

2. With the help of this proposed system the paper work is drastically reduced.

3. As this system is automatic, there is no need to manually give input to this system.

4. There is no need of extra hardware unlike fingerprint based system.

5. This system is affordable as well as portable.

\subsection{Applications}

1. Government organizations and various enterprises can make use of this system to mark the attendance of their employee.

2. This system can also be integrated in the CCTV of schools and colleges to keep track of the attendance.

3. Can also be used in various video conferencing applications such as Google meet, Zoom meeting.

\section{CONCLUSION}

In this system, an attendance system for a lecture, section, or laboratory, allowing the lecturer or teaching assistant to keep track of students' attendance. It will save time and effort, particularly if the lecture has a high number of students. To solve the drawbacks of the previous (manual) approach, the Automated Attendance Approach was developed.

This attendance system demonstrates the use of image processing techniques in theclassroom. This strategy has the ability to boost both an institution's reputation and attendance system. In future this system can be integrated with CCTV cameras or online meeting apps such as googlemeet, zoom meeting for attendance purpose. 


\section{REFERENCES}

[1] K. R. Pireva, J. Siqeca, and S. Berisha, "RFID: Management system for students' attendance," in IFAC Proceedings Volumes (IFACPapersOnline), 2013, vol. 15, no. PART 1, pp. 137-140.

[2] R. Lodha, S. Gupta, H. Jain, and H. Narula, "Bluetooth Smart based attendance management system," in Procedia Computer Science, 2015, vol. 45, no. C, pp. 524-527.

[3] N. D. Veer and B. F. Momin, "An automated attendance system using video surveillance camera," in 2016 IEEE International Conference on Recent Trends in Electronics, Information \& Communication Technology (RTEICT), 2016

[4] I. L. Ruiz and M. Á. Gómez-Nieto, "Combining of NFC, BLE and Physical Web Technologies for Objects Authentication on IoT Scenarios," in Procedia Computer Science, 2017, vol. 109, pp. 265-272.

[5] K. Mohammed, A. S. Tolba, and M. Elmogy, "Multimodal student attendance management system (MSAMS)," Ain Shams Eng. J., vol. 9, no. 4, pp. 29172929, Dec. 2018

[6] J. Qin, X. J. Shen, M. Zou, and S. P. Qin, "An Automotive Needle Meter Dynamic Test Method Based on Computer Vision and HIL Technology," in Procedia Computer Science, 2018, vol. 154, pp. 588-595.

[7] J. Zhang, L. He, M. Karkee, Q. Zhang, X. Zhang, and Z. Gao, I "Branch detection for apple trees trained in fruiting wall architecture using depth features and RegionsConvolutional Neural Network (R- CNN)," Comput. Electron. Agric., vol. 155, pp. 386-393, Dec. 2018.

[8] X. Liu, H. A. Ounifi, A. Gherbi, Y. Lemieux, and W. Li, "A hybrid GPU-FPGA-based computing platform for machine learning," in Procedia Computer Science, 2018, vol. 141, pp. 104-111.

[9] D. Sunaryono, J. Siswantoro, and R. Anggoro, "An android based course attendance system using face recognition," J. King Saud Univ. - Comput. Inf. Sci., 2019.

[10] M. SyaifulRomadhon, A. Rahmah, and Y. Wirani, "Blended learning system using social media for college student: A case of tahsin education," in Procedia Computer Science, 2019, vol. 161, pp. 160-167.

[11] S. V. Shavetov, I. I. Merkulova, A. A. Ekimenko, O. I. Borisov, and V. S. Gromov, "Computer Vision in Control and Robotics for $\backslash$ Educational Purposes," in IFAC-PapersOnLine, 2019, vol. 52, no. 9, pp. 144-146.

[12] de Vicente Mohino, Bermejo Higuera, Bermejo Higuera, and Sicilia Montalvo, "The Application of a New Secure Software Development Life Cycle (S-SDLC) with Agile Methodologies," electronics, vol. 8, no. 11, p. 1218, Oct. 2019.

[13] A. Winkler-Schwartz et al., "Artificial Intelligence in Medicall Education: Best Practices Using Machine Learning to Assess Surgical Expertise in Virtual Reality
Simulation," J. Surg. Educ., vol. 76, no. 6, pp. 16811690, Nov. 2019.

[14] G. Guo and N. Zhang, "A survey on deep learning based face recognition," Comput. Vis. Image Underst., vol. 189, Dec. 2019.

[15] WenxianZeng, QinglinMeng, Ran Li "Design of Intelligent Classroom Attendance System Based on Face Recognition” 2019 IEEE $3^{\text {rd }}$ Information Technology, Networking, Electronic and Automation Control Conference (ITNEC 2019)

[16] A. Elmahmudi and H. Ugail, "Deep face recognition using imperfect facial data," Futur.Gener.Comput. Syst., vol. 99, pp. 213-225, Oct. 2019.

[17] L. K. Almajmaie, O. N. Ucan, and O. Bayat, "Fingerprint recognition system based on modified multi-connect architecture (MMCA)," Cogn. Syst. Res., vol. 58, pp. 107-113, Dec. 2019.

[18] S. M. Bah and F. Ming, "An improved face recognition algorithm and its application in attendance management system," Array, vol. 5, p. 100014, Mar. 2020.

[19] U. Jayaraman, P. Gupta, S. Gupta, G. Arora, and K. Tiwari, "Recent development in face recognition," Neuro computing, 2020.

[20] S. Afra and R. Alhajj, "Early warning system: From face recognition by surveillance cameras to social media analysis to detecting suspicious people," Phys. A Stat. Mech. its Appl., vol. 540, p. 123151, Feb. 2020.

[21] I. Jegham, A. Ben Khalifa, I. Alouani, and M. A. Mahjoub, "Visionbased human action recognition: An overview and real world challenges," Forensic Sci. Int. Digit. Investig., vol. 32, p. 200901, Mar. 2020.

[22] D. X. Zhou, "Theory of deep convolutional neural networks: Down sampling," Neural Networks, vol. 124, pp. 319-327, Apr. 2020.

[23] M. You, X. Han, Y. Xu, and L. Li, "Systematic evaluation of deep face recognition methods," Neuro computing, vol. 388, pp. 144-156, May 2020.

[24] A. Khatami, A. Nazari, A. Khosravi, C. P. Lim, and S Nahavandi, "A weight perturbation-based regularisation technique for convolutional neural networks and the application in medical imaging," Expert Syst. Appl., vol. 149, Jul. 2020.

[25] R. Taufiq, M. Baharun, B. Sunaryo, B. Pudjoatmodjo, and W. M. Utomo, "Indonesia: Covid-19 and E-Learning in Student Attendance Method," SciTech Framew., vol. 2, no. 1, pp. 12-22, 2020.

[26] SonaliAppasahebPatil, L. Arun Raj, Bhupesh Kumar Singh, "Prediction of IoT Traffic Using the Gated Recurrent Unit Neural Network- (GRU-NN-) Based Predictive Model", Security and Communication Networks, vol. 2021, Article ID 1425732, 7 pages, 2021.

[27] S. Patil and L. A. Raj, "Classification of traffic over collaborative IoT and Cloud platforms using deep learning recurrent LSTM", csci, vol. 22, no. 3, Sep. 2021. 